\title{
Dielectric studies of nanocrystalline calcium tungstate
}

\author{
N. Aloysius ${ }^{1 *}$, M.S. Rintu ${ }^{2}$, E. M. Muhammed ${ }^{2}$, T. Varghese $^{1,3}$ \\ ${ }^{1}$ Department of Physics, Newman College Thodupuzha-685 585, Kerala, India \\ (Affiliated to M. G. University, Kottayam) \\ ${ }^{2}$ Department of Physics, Maharajas College, Ernakulam-682 011, Kerala, India \\ ${ }^{3}$ Nanoscience Research Centre (NSRC), Department of Physics, Nirmala College, \\ Muvattupuzha - 686 661, Kerala, India \\ *nanoncm@gmail.com
}

\begin{abstract}
Nanocrystalline samples of $\mathrm{CaWO}_{4}$ were prepared at room temperature by simple chemical precipitation. The samples were characterized by X-ray diffraction and scanning electron microscopy. Energy dispersive X-ray analysis confirmed the elements present in the sample. The frequency and temperature dependence of the dielectric constant and ac electrical conductivity of the nanomaterial were investigated. Very low dielectric loss in nanocrystalline $\mathrm{CaWO}_{4}$ powder was observed at high frequencies. The values of ac electrical conductivity calculated from the permittivity studies were found to increase as frequency increased, conforming to small polaron hopping.
\end{abstract}

Keywords: Chemical precipitation, dielectric constant, ac electrical conductivity, polaron hopping.

Received: 29 January 2016

Revised: 21 May 2016

\section{Introduction}

Nanocrystalline $\mathrm{CaWO}_{4}$ has attracted particular interest because of its practical applications, such as laser host materials in quantum electronics and scintillators in medical devices [1-6]. It has been reported that $\mathrm{CaWO}_{4}$ of scheelite-like structures is an excellent blue-emitting phosphor by their radiation of ultraviolet (UV) light [7]. Also, $\mathrm{CaWO}_{4}$ has shown considerable promise as a fiber-matrix interlayer in oxide ceramic composites [8]. The lower dielectric constant and low loss make nanostructured $\mathrm{CaWO}_{4}$ a promising candidate for applications as a low temperature co-fired ceramic (LTCC), substrate, and electronic packaging material [9]. Pullar et al. explained the microwave dielectric properties of $\mathrm{AWO}_{4}(\mathrm{~A}=\mathrm{Mg}, \mathrm{Zn}, \mathrm{Ni}$ and $\mathrm{Co})$ compounds with extrinsic parameter, such as density [10]. Sreedevi et al. reported that $\mathrm{Ag}_{2} \mathrm{WO}_{4}$ nanoparticles can be a promising material for the high dielectric constant gate in Si-based complementary metal oxide semiconducting devices [11]. The influence of processing methods on the characteristics of $\mathrm{CdWO}_{4}$ powders and the related microwave dielectric properties were reported by Bao-Chun Guo et al. [12]. The study of dielectric properties of samples as a function of temperature and frequency may help in identifying their potential applications [13]. The characterization of dielectric behavior is very important not only to the theory of the polarization mechanism but also from an application point of view, where knowledge of the temperature and the frequency dependence of dielectric constant are very important. The relative dielectric constant of the material determines its ability to store electrostatic energy.

Dielectric studies of $\mathrm{CaWO}_{4}$ nanoparticles are incomplete and need further investigation. In the present work, we synthesized $\mathrm{CaWO}_{4}$ nanoparticles by chemical precipitation followed by calcination. The samples were then characterized by X-ray diffraction (XRD) and scanning electron microscopy (SEM). The frequency and temperature dependence of dielectric properties of sintered pellets made out of the products were then investigated.

\section{Materials and methods}

Calcium nitrate $\mathrm{Ca}\left(\mathrm{NO}_{3}\right)_{2} \cdot 4 \mathrm{H}_{2} \mathrm{O}\left(99.8 \%\right.$, Sigma Aldrich) and sodium tungstate $\left.\mathrm{Na}_{2} \mathrm{WO}_{4} \cdot 2 \mathrm{H}_{2} \mathrm{O}\right)(99.9 \%$, Alfa Aesar) analytical grade reagents were used for the preparation of $\mathrm{CaWO}_{4}$ nanocrystals. The samples were prepared by reacting aqueous solutions of calcium nitrate and sodium tungstate $(0.1 \mathrm{M}$ each) at room temperature. The precipitate formed was centrifuged, filtered, washed with distilled water a number of times, and dried in an oven to get fine powders of calcium tungstate. $\mathrm{S}_{1}$ and $\mathrm{S}_{2}$ are samples of nanocrystalline $\mathrm{CaWO}_{4}$ were calcined at 650 and $750{ }^{\circ} \mathrm{C}$, respectively. XRD studies of these samples were conducted using Bruker D8 Advance X-ray diffractometer $(\lambda=1.5406 \AA)$ with $\mathrm{CuK} \alpha$ radiation in $2 \theta$ range from 20 to $80^{\circ}$. The morphological analysis of $\mathrm{CaWO}_{4}$ nanoparticles was carried out with a scanning electron microscope JEOL MODEL JSM-6390LV, operating at $20 \mathrm{kV}$ measurements. 
The calcined powder sample was cold pressed in the form of cylindrical pellets of diameter $13 \mathrm{~mm}$ and thickness $d \sim 1.5 \mathrm{~mm}$ by applying a pressure of $\sim 10 \mathrm{GPa}$ using a hand operated hydraulic press. The pellets were then sintered at $500{ }^{\circ} \mathrm{C}$. The density of the pellet was determined to be $4.88 \mathrm{~g} / \mathrm{cm}^{3}$. The circular faces of the pellets were made electrically conducting by coating with silver paste. Dielectric measurements as a function of frequency in the range of $100 \mathrm{~Hz}-1 \mathrm{MHz}$ were measured at various selected temperatures from $303-423 \mathrm{~K}$ using an LCR meter (Wayne Kerr H-6500 model) in conjunction with a portable furnace and temperature controller $( \pm 1 \mathrm{~K})$.

\section{Results and discussion}

The powder XRD spectra of $\mathrm{CaWO}_{4}$ nanoparticle samples are shown in Fig. 1. Both the samples showed characteristic peaks of scheelite structure with tetragonal unit cell. The ' $\mathrm{d}$ ' values taken from the JCPDS file No. 77-2235 for $\mathrm{CaWO}_{4}$ are in close agreement with the observed 'd' values.

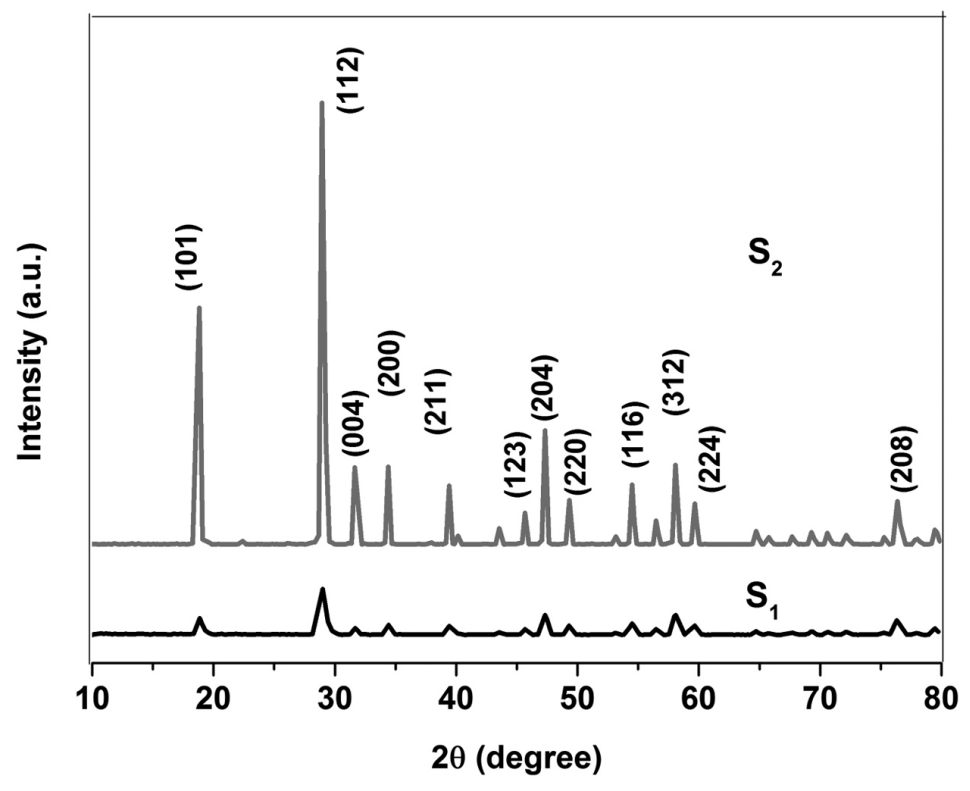

FIG. 1. XRD spectra of $\mathrm{CaWO}_{4}$ samples

In general, the nanocrystallite size can be estimated from the Scherrer's formula: $D_{h k l}=K \lambda /(\beta \cos \theta)$, where $\lambda$ is the $\mathrm{x}$-ray wavelength $(0.15405 \mathrm{~nm}), \beta$ the full-width at half maximum, $\theta$ the diffraction angle, $K$ is a constant $(0.89)$ and $D_{h k l}$ the size along the $(h k l)$ direction. From the analysis, the average crystallite size obtained was $39 \mathrm{~nm}$ for $\mathrm{S}_{1}$ and $44 \mathrm{~nm}$ for $\mathrm{S}_{2}$.

The SEM image of $\mathrm{CaWO}_{4}$ nanoparticles calcined at $650{ }^{\circ} \mathrm{C}$ is shown in Fig. 2(a). They are clusters shaped like dumb-bells. The elemental analysis of the sample $\mathrm{S}_{1}$ was done by energy dispersive X-ray (EDX) spectroscopy. Fig. 2(b) shows typical EDX spectrum of synthesized $\mathrm{CaWO}_{4}$ nanoparticles. The peaks of the spectrum confirmed that the product contains $\mathrm{Ca}, \mathrm{W}$ and $\mathrm{O}$. The intense signal near at $1.774 \mathrm{keV}$ indicates that $\mathrm{W}$ is the major element.

The dielectric constant and $a c$ conductivity $\left(\sigma_{a c}\right)$ were calculated by using equations $\varepsilon^{\prime}=C d / \varepsilon_{0} A$ and $\sigma_{a c}=\varepsilon^{\prime} \varepsilon_{0} \omega \tan \delta$, respectively, where $A$ is the face area, $C$ the measured capacitance of the pellet, $\varepsilon_{0}$ the permittivity of vacuum, $\omega$ the angular frequency and $\tan \delta$ the loss tangent. Fig. 3(a) shows the variation of dielectric constant with frequency for temperatures from 303 to $423 \mathrm{~K}$ of sample $\mathrm{S}_{1}$. It is seen that the dielectric constant for all temperatures are high at low frequencies which decreased rapidly as frequency increased, attaining a constant value at higher frequencies. For $303 \mathrm{~K}$, the value of $\varepsilon$ was 24.74 at $100 \mathrm{~Hz}$, which decreased to 7.11 at $1 \mathrm{MHz}$. At $393 \mathrm{~K}$, the values were $30.08(100 \mathrm{~Hz})$ and $7.15(1.0 \mathrm{MHz})$. The corresponding values for $423 \mathrm{~K}$ were 39.54 at $100 \mathrm{~Hz}$ and 7.20 at $1.0 \mathrm{MHz}$. Fig. 3(b) shows a similar variation for samples $\mathrm{S}_{1}$ and $\mathrm{S}_{2}$, at $393 \mathrm{~K}$. At lower frequencies the dielectric constant is found to be higher for the sample having smaller grain size $\left(\mathrm{S}_{1}\right)$, but approaches a constant value beyond $0.1 \mathrm{MHz}$. When temperature is increased, more and more dipoles are oriented, resulting in an increase in the dielectric constant for a given value of frequency [14]. At very high frequencies $(\mathrm{MHz})$, the charge carriers would have started to move before the field reversal occurs and $\varepsilon^{\prime}$ falls to a small value at higher frequencies. 


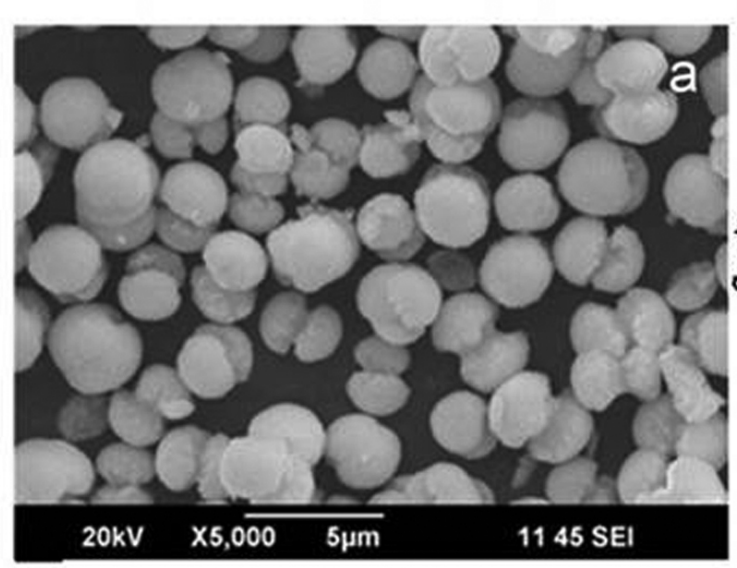

FIG. 2. (a) SEM image of $\mathrm{CaWO}_{4}\left(\mathrm{~S}_{1}\right)$ and (b) EDX spectrum of $\mathrm{CaWO}_{4}$
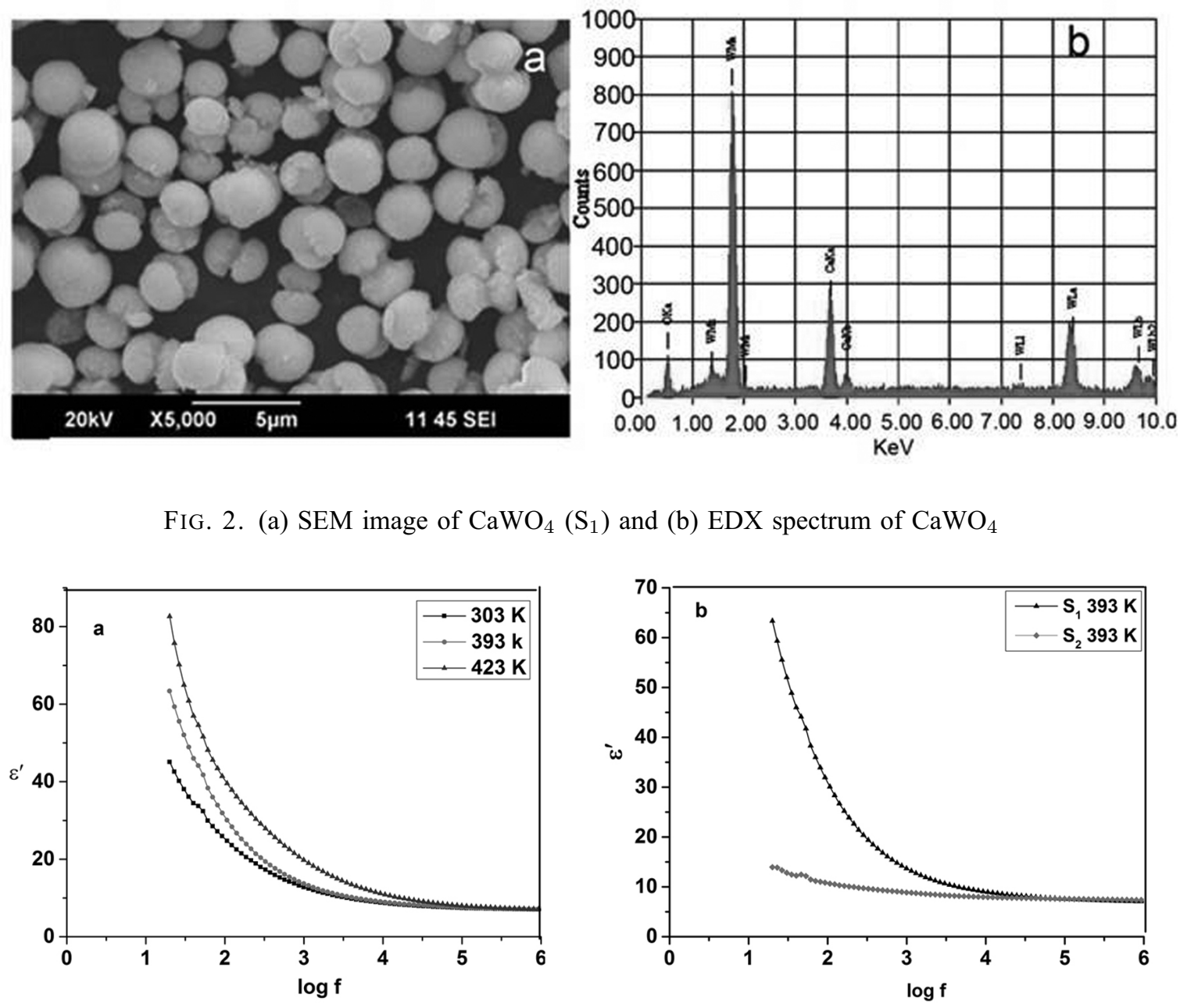

FIG. 3. The variation of dielectric constant with frequency of (a) sample $\mathrm{S}_{1}$ at temperatures 303 , 393 and $423 \mathrm{~K}$ and (b) samples $\mathrm{S}_{1}$ and $\mathrm{S}_{2}$ at $393 \mathrm{~K}$

Space charge polarization and reversal of the polarization direction contributes much to the $\varepsilon^{\prime}$ [15]. With the increase in volume of the particle, the volume of the interfaces decreases. When volume increases, the contribution to $\varepsilon^{\prime}$ by electronic relaxation polarization inside the particles increases.

The frequency dependence of dielectric loss of sample $S_{1}$ is shown in Fig. 4(a). The loss factor represented by $\tan \delta$ has a value of 3.26 at $100 \mathrm{~Hz}$ which decreases slowly to zero at higher frequencies. At $393 \mathrm{~K}$, the corresponding variation is not very different. For $423 \mathrm{~K}, \tan \delta$ has a value of 9.53 at $100 \mathrm{~Hz}$ which gradually decreases almost to 0 at frequencies beyond $0.10 \mathrm{MHz}$. At $393 \mathrm{~K}$ the corresponding variation is not very different. The decrease in $\tan \delta$ takes place when the jumping rate of charge carriers lags behind the alternating electric field beyond a certain critical frequency. The inhomogeneities present in the interface layers in $\mathrm{CaWO}_{4}$ nanocrystals produce an absorption current resulting in dielectric loss. This absorption current decreases with increase in frequency of the applied field. The hopping probability per unit time increases with increase in temperature. Correspondingly, the loss tangent also increases with increase of temperature [16]. The variation of $\tan \delta$ with frequency at $393 \mathrm{~K}$ for samples $\mathrm{S}_{1}$ and $\mathrm{S}_{2}$ with different grain sizes is shown in Fig. 4(b). At $100 \mathrm{~Hz}$, the value is 3.4 for $\mathrm{S}_{1}$ which decreases to 0.9 for $\mathrm{S}_{2}$. This variation of $\tan \delta$ for different grain sizes is due to size effect [17]. The low value of $\tan \delta$ indicates its potential for microwave applications.

The loss in $\mathrm{CaWO}_{4}$ can be explained using the electronic hopping model, which considers the frequency dependence of the localized charge carriers hopping in a random array. This model is applicable for materials in which the polarization responds rapidly to the appearance of an electron on any one site so that the transition may be said to occur effectively into the final state [17]. In the high frequency region tan $\delta$ becomes almost zero because the electron exchange interaction (hopping) cannot follow the alternatives of the applied ac electric field beyond a critical frequency. 

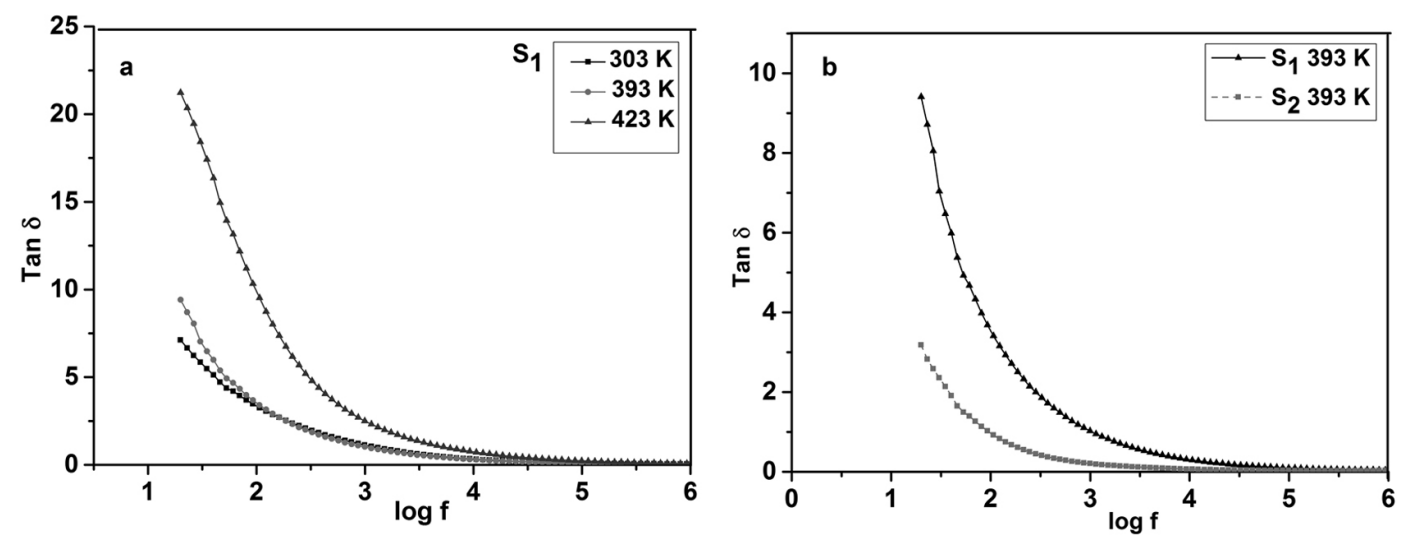

FIG. 4. The variation of loss tangent with frequency of (a) sample $\mathrm{S}_{1}$ at temperatures 303,393 and $423 \mathrm{~K}$ and (b) samples $\mathrm{S}_{1}$ and $\mathrm{S}_{2}$ at $393 \mathrm{~K}$

Figure 5(a) shows the variation of ac conductivity $\left(\sigma_{a c}\right)$ of sample S1with frequency. Initially, it has a small value which increased at higher frequencies. The nature of variation is similar for other temperatures, but the values are shifted upwards as the temperature is raised. For $303 \mathrm{~K}, \sigma_{a c}$ has a value of $5.001 \times 10^{-7} \mathrm{~S} / \mathrm{m}$ at $100 \mathrm{~Hz}$ which increased slowly at higher frequencies to $1.4 \times 10^{-3} \mathrm{~S} / \mathrm{m}$ at $0.10 \mathrm{MHz}$. At $393 \mathrm{~K}$, the corresponding variation was not very different. For $423 \mathrm{~K}$, the values were $2.236 \times 10^{-6} \mathrm{~S} / \mathrm{m}$ at $100 \mathrm{~Hz}$ and $2.781 \times 10^{-5} \mathrm{~S} / \mathrm{m}$ at $1.0 \mathrm{MHz}$. The variation of $\sigma_{a c}$ with frequency at $393 \mathrm{~K}$ for different grain sizes is shown in Fig. 5(b). At $100 \mathrm{~Hz}, \sigma_{a c}$ is found to be $5.002 \times 10^{-7} \mathrm{~S} / \mathrm{m}$ for $\mathrm{S}_{1}$ which increased to $6.88 \times 10^{-5} \mathrm{~S} / \mathrm{m}$ at $1.0 \mathrm{MHz}$. For $\mathrm{S}_{2}$, the corresponding values were $5.823 \times 10^{-8} \mathrm{~S} / \mathrm{m}$ and $7.671 \times 10^{-6} \mathrm{~S} / \mathrm{m}$. It is clear from the figure that the conductivity increased as frequency increased conforming to small polaron hopping [18]. Also, there is a possibility of conduction due to impurities at low temperature. It is found that at given temperature and frequency, $\sigma_{a c}$ is higher for particle having smaller size. According to Elliot's barrier hopping model, ac conductivity increases with hopping distance [19]. Therefore, it may be concluded that in $\mathrm{CaWO}_{4}$ hopping distance increased with reduction in particle size.
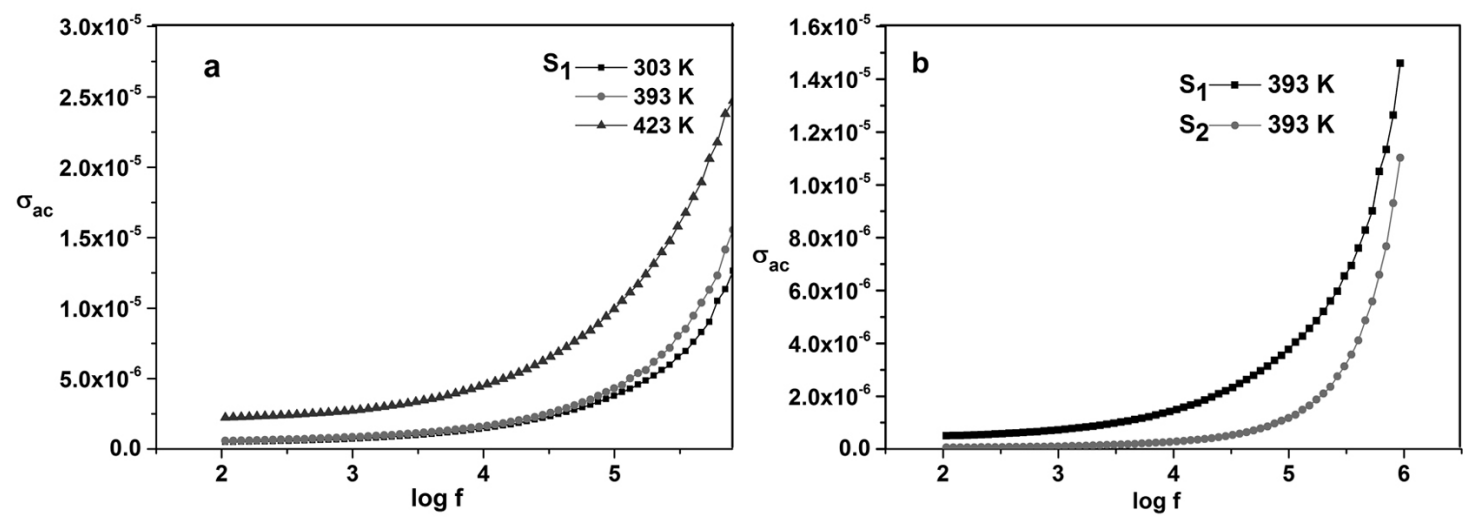

FIG. 5. The variation of ac electrical conductivity with frequency of (a) sample $\mathrm{S}_{1}$ at temperatures 303, 393 and $423 \mathrm{~K}$; (b) samples $\mathrm{S}_{1}$ and $\mathrm{S}_{2}$ at $393 \mathrm{~K}$

\section{Conclusion}

The $\mathrm{CaWO}_{4}$ nanoparticles were prepared at room temperature by simple chemical precipitation reaction without any catalyst, surfactant, or templates. The dielectric properties of $\mathrm{CaWO}_{4}$ were determined as a function of frequency from $100 \mathrm{~Hz}$ to $1.0 \mathrm{MHz}$ for temperatures ranging from 303 to $423 \mathrm{~K}$. At lower frequencies, $\varepsilon^{\prime}$ and $\tan \delta$ have higher values while at higher frequencies the values reached steady lower values. Similar variation was observed when the temperature was raised but the values of $\varepsilon^{\prime}$ and $\tan \delta$ were elevated. The ac conductivity increased as frequency was increased conforming to small polaron hopping. The values of $\varepsilon^{\prime}$, $\tan \delta$ and $\sigma_{a c}$ showed considerable increase as the particle size was reduced. The very low value of loss tangent obtained for 
$\mathrm{CaWO}_{4}$ nanocrystals suggests that it is potentially useful for microwave applications. It was found that the applied frequency, temperature and particles size affect the dielectric properties of the $\mathrm{CaWO}_{4}$ nanocrystals.

\section{Acknowledgements}

The authors are indebted to NSRC, Nirmala College, Muvattupuzha and Newman College, Thodupuzha for the support to undertake this study. The financial support from the University Grants Commission, New Delhi, India (FIP/12th Plan/KLMG020 TF03) is greatly acknowledged.

\section{References}

[1] Kobayashi M., Ishii M., Usuki Y., Yahagi H. Scintillation characteristics of PbWO4 single crystals at room temperature. Nuclear Instruments and Methods in Physics Research A, 1993, 333 (2-3), P. 429-433.

[2] Katelnikovas A., Grigorjeva L., et al. Sol-Gel Preparation of Nanocrystalline $\mathrm{CaWO}_{4}$. Lithuanian J. of Physics, 2007,47 (1), P. 63-68.

[3] Phuruangrat A., Thongtem T., Thongtem S. Synthesis, characterization and photoluminescence of nanocrystalline calcium tungstate. J. Exp. Nanosci., 2009, 5, P. 263-267.

[4] Lou Z., Cocivera M. Cathodoluminescence of $\mathrm{CaWO}_{4}$ and SrWO4 thin films prepared by spray pyrolysis. Mater. Res. Bull., 2002, 37, P. 1573-1582.

[5] Nagirnyi V., Feldbach E., et al. Excitonic and recombination processes in $\mathrm{CaWO}_{4}$ and $\mathrm{CdWO}_{4}$ scintillators under synchrotron irradiation. Radiat. Meas., 1998, 29, P. 247-250.

[6] Oishi S., Hirao M. Growth of $\mathrm{CaWO}_{4}$ whiskers from $\mathrm{KCl}$ flux. J.Mater.Sci., Lett., 1989, 8 (12), P. $1397-1398$.

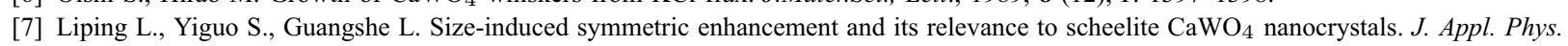
Lett., 2007, 90, P. 054105-054107.

[8] Mogilevsky P., Parthasarathy T.A., Petry M.D. Anisotropy in room temperature microhardness and fracture of CaWO 4 scheelite. Acta Materialia, 2004, 52, P. 5529-5537.

[9] Vidya S., Sam Solomon., Thomas J.K. Synthesis of Nanocrystalline $\mathrm{CaWO}_{4}$ as Low-Temperature Co-Fired Ceramic Material: Processing, Structural and Physical Properties. Journal of Electronic Materials, 2012, 42, P. 129-137.

[10] Pullar R.C., Farrah S., Alford N.M. $\mathrm{MgWO}_{4}, \mathrm{ZnWO}_{4}, \mathrm{NiWO}_{4}$ and $\mathrm{CoWO}_{4}$ microwave dielectric ceramics. Journal of the European Ceramic Society, 2007, 27, P. 1059-1063.

[11] Sreedevi A., Priyanka K.P., et al. Nanophase $\alpha$-Silver Tungstate for Potential Applications in Light Emitting Diodes and Gate Dielectrics. Advanced Science, Engineering and Medicine, 2015, 7, P. 498-505.

[12] Bao-Chun Guo, Peng Liu, et al. Effect of Preparation Methods on Microstructures and Microwave Dielectric Properties of CdWO 4 ceramics. Integrated Ferroelectrics, 2015, 167, P. 107-114.

[13] Chi Kao K. Dielectric phenomena in Solids. Elsevier, New York, 2004.

[14] Kar T., Choudhary R.N., Sharma S., Singh K.S. Structural and electrical properties of $\mathrm{Ba}_{2} \mathrm{Na}_{3} \mathrm{RNb}_{10} \mathrm{O}_{30}$ Ceramics. Indian J. Phys. A, 1999, 73 (4), P. 453-459.

[15] Ravinder D., Vijayakumar K. Dielectric behaviour of erbium substituted Mn-Zn ferrites. Bull. Mater. Sci., 2001,24 (5), P. $505-509$.

[16] Potty S.N., Khader M.A. Dielectric Properties of nanophase $\mathrm{Ag}_{2} \mathrm{HgI}_{4}$ and $\mathrm{Ag}_{2} \mathrm{HgI}_{4}-\mathrm{Al}_{2} \mathrm{O}_{3}$ nanocomposites. Bull. Mater. Sci., 2000, 23 (5), P. 361-367.

[17] Murthy V., Sobhanadri J. Dielectric properties of some nickel-zinc ferrites at radio frequency. Phys Status Solidi (a), 1976, 36 (2), K133-K135.

[18] Priyanka K.P., Sunny J., et al. Dielectric properties and a. c. conductivity of nanocrystalline titania. J. Basic Appl. Phys., 2013, 2, P. $105-108$.

[19] Elliot S.R. Temperature dependence of a.c. conductivity of chalcogenide glasses. Phil. Mag. Part B, 1978,37 (5), P. 553. 